\title{
A high sensitivity fiber Bragg grating seismic sensor system for intrusion detection
}

\section{Zhihui Sun, Meng Wang, Shujuan Li, Li Min, Yuanyuan Yang, et al.}

Zhihui Sun, Meng Wang, Shujuan Li, Li Min, Yuanyuan Yang, Jiasheng Ni, Chang Wang, "A high sensitivity fiber Bragg grating seismic sensor system for intrusion detection," Proc. SPIE 11144, Photonics and Education in Measurement Science 2019, 111440I (17 September 2019); doi: $10.1117 / 12.2532728$

EDIE Event: Joint TC1 - TC2 International Symposium on Photonics and Education in Measurement Science 2019, 2019, Jena, Germany 


\title{
A High Sensitivity Fiber Bragg Grating Seismic Sensor System for Intrusion Detection
}

\author{
Zhihui Sun ${ }^{*}$, Meng Wang, Shujuan Li, Li Min, Yuanyuan Yang, Jiasheng Ni, Chang Wang \\ Laser Institute, Qilu University of Technology (Shandong Academy of Sciences), Jinan, China
}

\begin{abstract}
In this paper we report the development and test of a high sensitivity fiber Bragg grating (FBG) seismic sensor system for intrusion detection application. A high sensitivity FBG seismic sensor is designed and its sensitivity is above $1000 \mathrm{pm} / \mathrm{g}$. Unbalanced Michelson interferometer and Phase generated carrier (PGC) algorithm are adopted by the demodulation system. The system noise is below $10^{-3} \mathrm{pm} / \sqrt{\mathrm{Hz}}$ and the minimum detectable seismic signal is below $1 \mu \mathrm{g} \sqrt{\mathrm{Hz}}$. The FBG seismic sensor has a maximum detection range of about 70 meters for human and about 200 meters for small wheeled vehicle.
\end{abstract}

Keywords: Fiber bragg grating; Seismic sensor; Intrusion detection; Field test

\section{INTRODUCTION}

In recent years, fiber optic seismic sensors have attracted considerable research interest ${ }^{[1-4]}$. This is due to their advantages of low noise floor, high sensitivity, and large dynamic range, immunity to electromagnetic interference, extreme robustness and reliability. Their application in intrusion detection, oil and gas exploration, structural health monitoring is attracting more and more interest.

In this paper we report the development and field test of a very high sensitivity fiber Bragg grating (FBG) geophone for intrusion detection application.

The paper is arranged as follows. Section 2 describes the principles of FBG seismic sensor system. Section 3 describes the performance of FBG seismic sensor system. Section 4 describes the test and results. Then the conclusion and acknowledgments is given.

\section{PRINCIPLES OF FBG SEISMIC SENSOR SYSTEM}

Principle of FBG seismic sensor sensing is illustrated in Fig. 1. The mass is mounted at the end of the L type cantilever beam as the inertia sensing component. The fiber grating is pre-stretched over the cantilever beam. When the FBG seismic sensor sense the seismic signal, the mass moves relative to the housing, the FBG is stretched or shrunk, resulting in the strain change and then the reflection wavelength of the FBG is changed. The wavelength shift $\Delta \lambda$ is proportion to the strain change $\Delta \varepsilon$,

$$
\frac{\Delta \lambda}{\lambda}=0.78 \cdot \Delta \varepsilon
$$

where $\lambda$ is the FBG wavelength, the factor 0.78 accounts for stress-optic coefficient. 


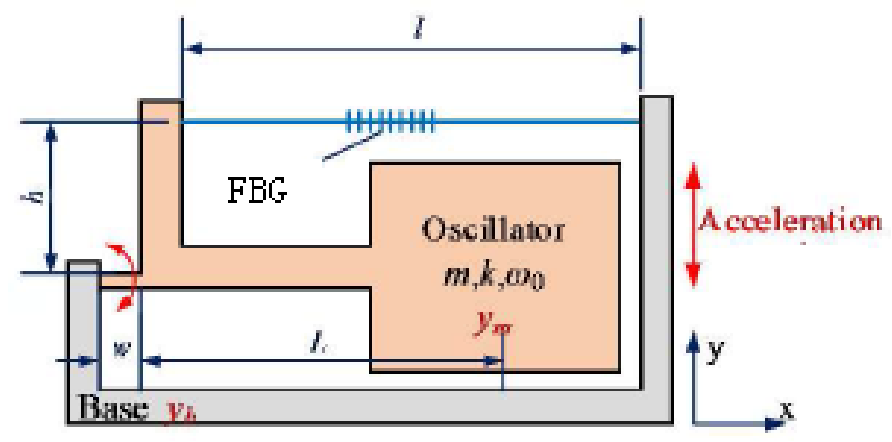

Fig. 1. Principle of FBG seismic sensing.

Schematic diagram of FBG seismic sensor system is illustrated in Fig. 2. An Amplified spontaneous emission (ASE) source is used, and its spectral width is 40nm. 16 FBG seismic sensors are wavelength division multiplexed from C62 to $\mathrm{C} 17$, and the wavelength interval is $2.4 \mathrm{~nm}$.

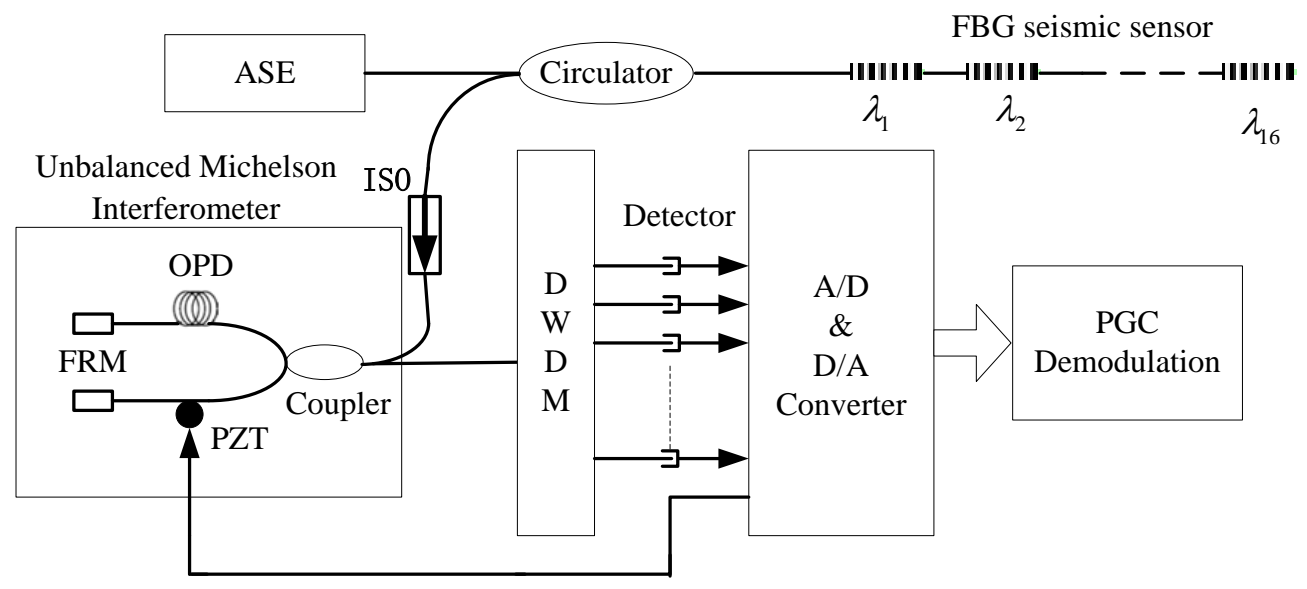

Fig. 2. Schematic diagram of FBG seismic sensor system.

The output of the FBG seismic sensors are injected into an unbalanced Michelson interferometer (MI). Then the wavelength shift $\Delta \lambda$ of the fiber laser sensor is converted into phase shift, the interferometer phase shift $\Delta \phi$ is given by

$$
\Delta \phi=\frac{4 \pi n L}{\lambda^{2}} \Delta \lambda
$$

where $L$ is the fiber path imbalance in the MI $(L=5 \mathrm{~mm}), n$ is the effective refractive index of the interferometer fiber. To eliminate the signal-fading caused by the initial phase shifts of the MI, a $5 \mathrm{kHz}$ sinusoidal voltage signal is applied to the piezoelectric tube (PZT) cylinder in one arm of the MI, then the phase modulation carrier is generated.

Dense wavelength division de-multiplexer (DWDM) is used to split off the MI output signals. The outputs of the DWDM are received by InGaAs PIN photodiode detection circuits. The analog outputs of the circuits are sampled by multi-channel A/D converter with 24bit resolution and 50kSPS. At the same time, the $5 \mathrm{kHz}$ PGC carrier signal generated by D/A converter is also sampled. The digital PGC demodulation algorithm as shown in Fig.3 is adopted to recover the signals $\Delta \phi(t)$. 


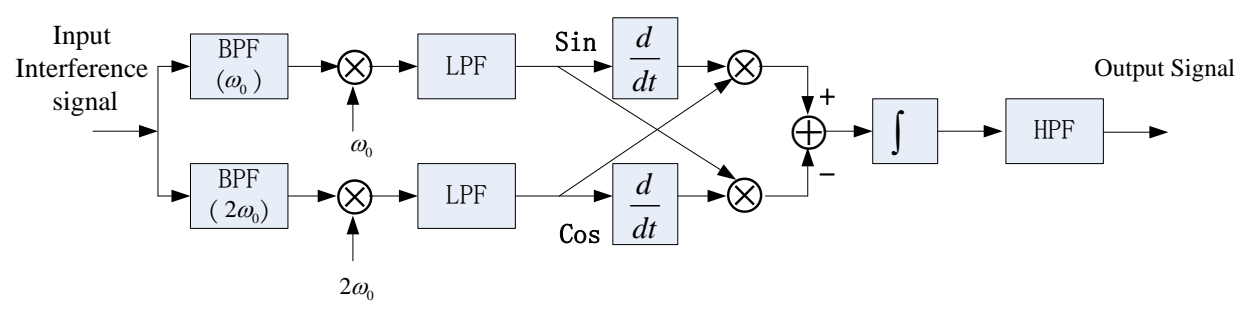

Fig. 3. Schematic diagram of digital PGC demodulation algorithm.

\section{PERFORMANCE OF FBG SEISMIC SENSOR SYSTEM}

The photo of two packaged FBG seismic sensor is illustrated in Fig. 4. The frequency response of the FBG seismic sensor is tested and the result is shown in Fig. 5. The sensitivity of the FBG seismic sensor is above 1000pm/g.

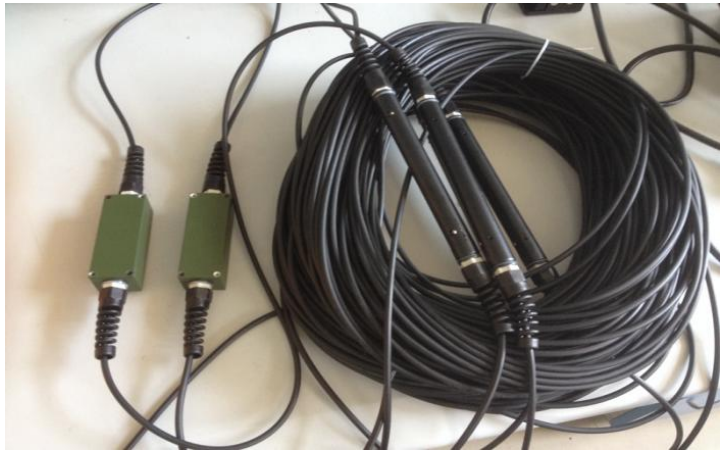

Fig. 4. The photo of packaged seismic sensors.

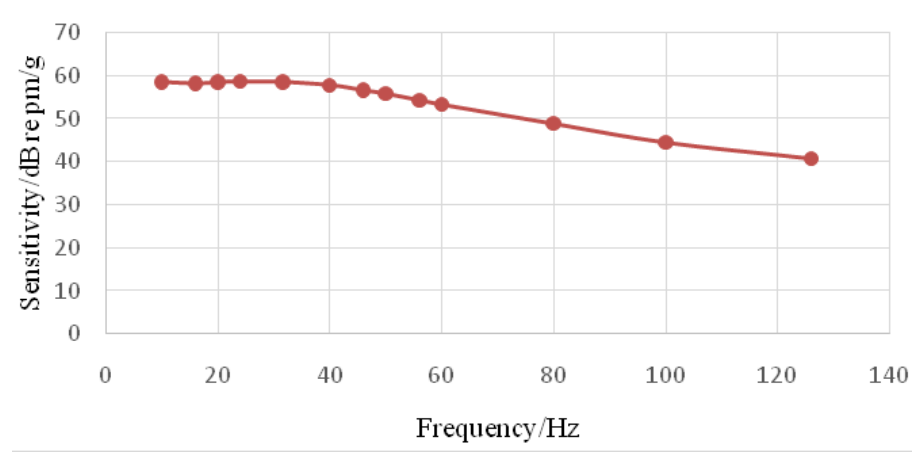

Fig. 5. Frequency response of a FBG seismic sensor.

The system and noise level of FBG seismic sensor in the frequency range from $3 \mathrm{~Hz}$ to $500 \mathrm{~Hz}$ is shown in Fig. 6. It can be observed that the system noise level is about $10^{-3} \mathrm{pm} / \sqrt{\mathrm{Hz}}(60 \mathrm{~dB}$ re $\mathrm{pm})$.
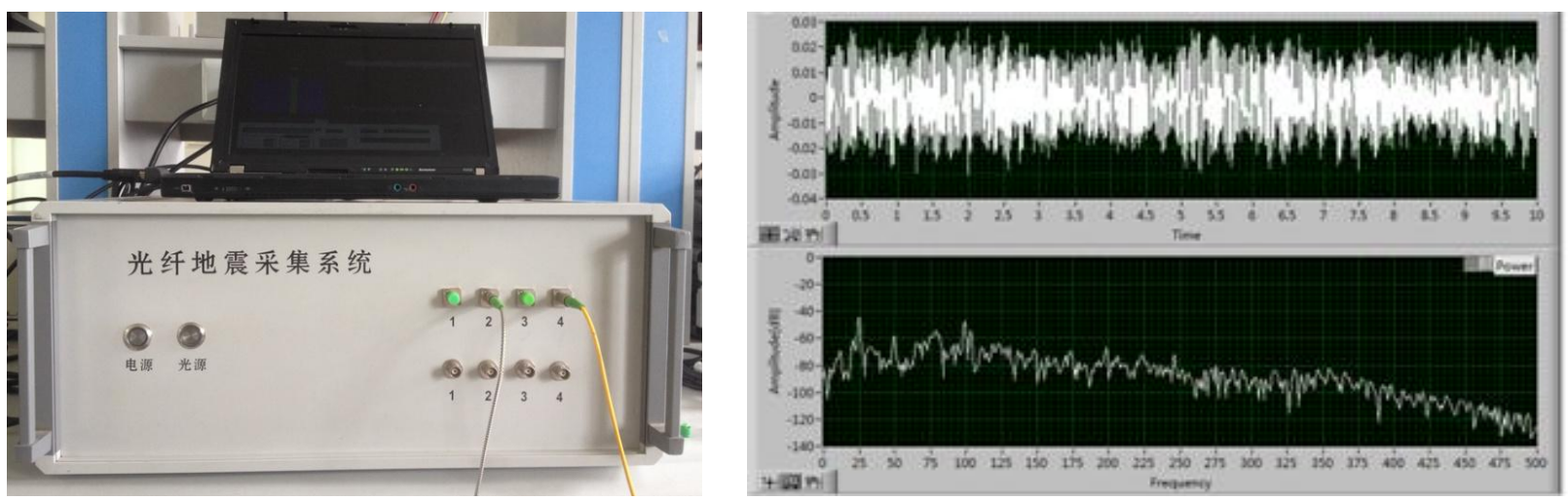

Fig. 6. The system and noise level of FBG seismic sensor.

The noise equivalent acceleration (NEA) is obtained as

$$
N E A=\frac{\phi_{n}(f)}{S_{a}(f)}
$$

where $\phi_{n}(f)$ is the noise floor of the FBG seismic sensor system, $S_{a}(f)$ is sensitivity of FBG seismic sensor. From Fig.5 and Fig.6, the acceleration sensitivity is $1000 \mathrm{pm} / \mathrm{g}$ and the noise floor is $10^{-3} \mathrm{pm} / \sqrt{\mathrm{Hz}} @ 40 \mathrm{~Hz}$. According to Eq. (3), the noise equivalent acceleration is $1 \mu \mathrm{g} / \sqrt{\mathrm{Hz}} @ 40 \mathrm{~Hz}$. 


\section{TEST AND RESULT}

In Laboratory of vibration and shock of National Institute of Measurement and Testing Technology, which located in Weihai City of Shandong Province, the performance of FBG seismic sensors are tested together with current state-of-the art coil and MEMS geophones. Frequency response, linearity, sine and pulse signal response of 3C fiber laser and other geophones are tested simultaneously on the shaker. Noise level and small sine signal response are tested simultaneously.

As can be seem from Fig.7, The lab test results show that the FBG seismic sensor has significantly better performance in very small sine signal detection, and it is crucial for weak micro-seismic signal detection.

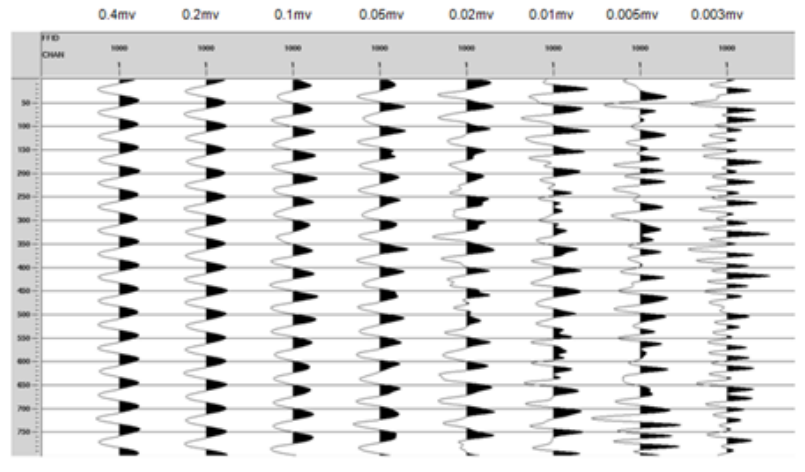

(a) FBG seismic sensor

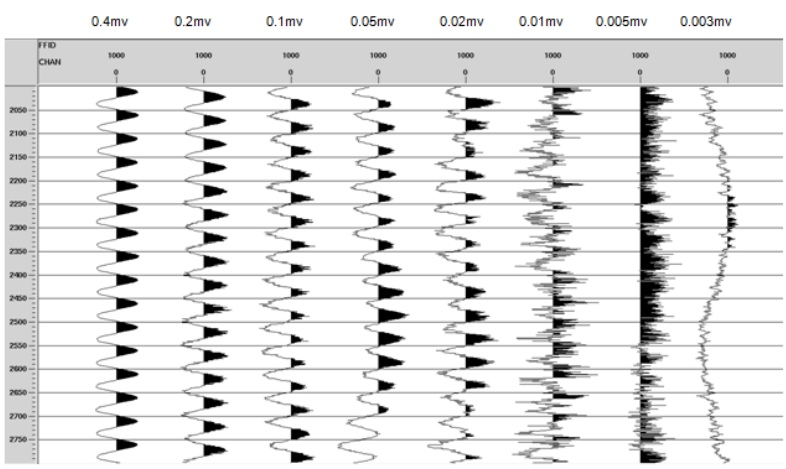

(c) Fiber optic interferometer seismic sensor

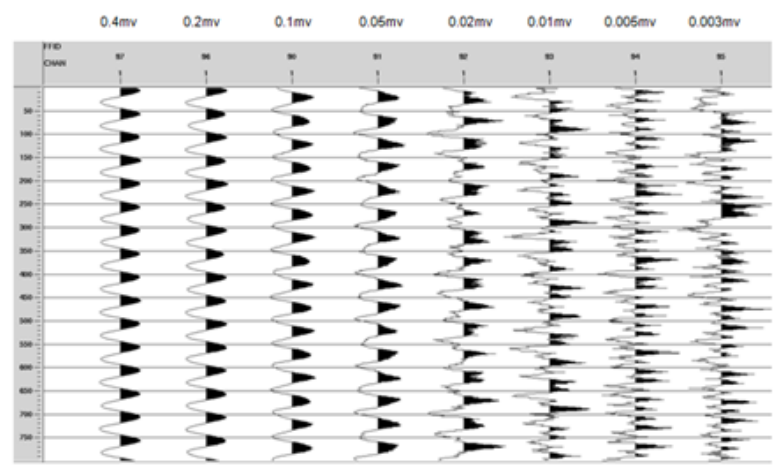

(b) Coil seismic sensor

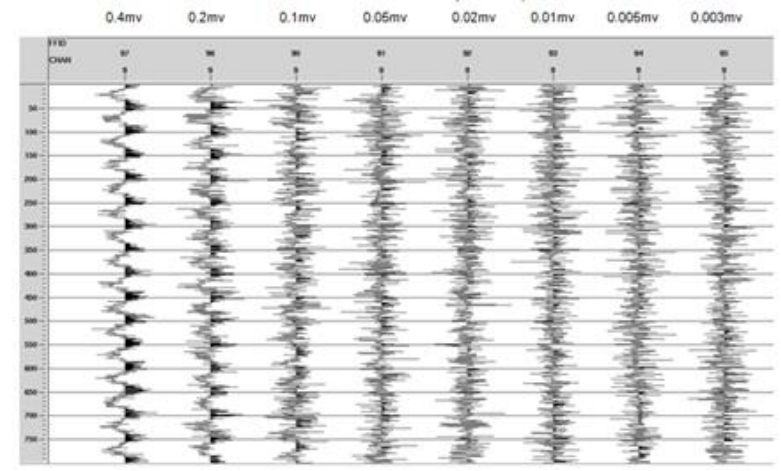

(d) MEMS seismic sensor

Fig.7 Small sine signal responses of seismic sensor (@20Hz).

The field test is performed to verify the performance of FBG seismic sensor system. Eight FBG seismic sensors are shallow buried with a depth of $10 \mathrm{~cm}$ and the space between them is $50 \mathrm{~m}$. Test target includes a human (weight: $80 \mathrm{~kg}$ ) and a small wheeled vehicle (weight: $1500 \mathrm{~kg}$ ). The human walks at a speed of 4 to $5 \mathrm{~km} / \mathrm{h}$. The signal waveforms of FBG seismic sensor for human walking at different distances are shown in Fig.8. The vehicle travels at a speed of $20 \mathrm{~km} / \mathrm{h}$. The signal waveform of FBG seismic sensor for the vehicle at 200m distances is shown in Fig 9. The FBG seismic sensor has a maximum detection range of about 70 meters for human and about 200 meters for small wheeled vehicle. 

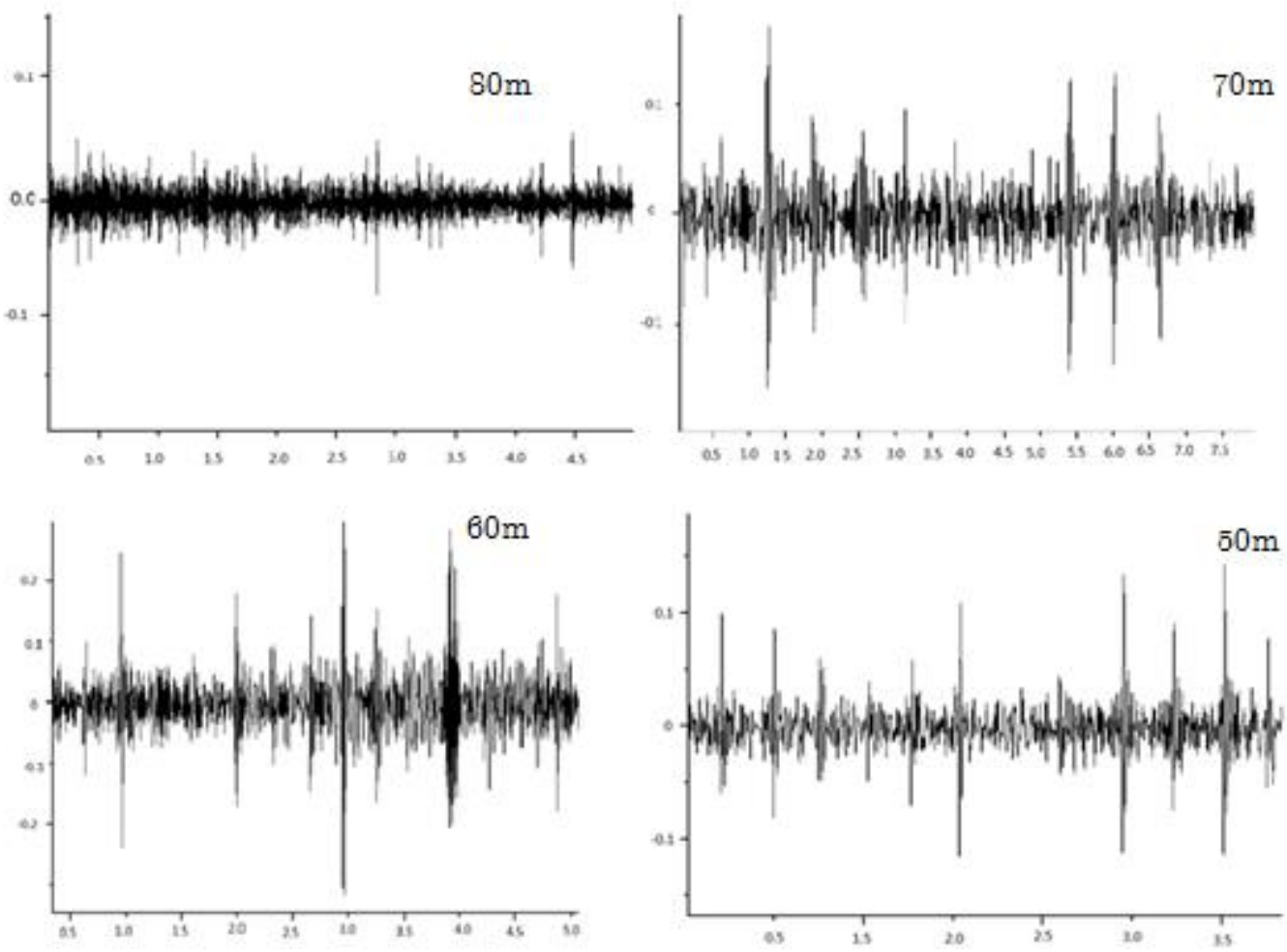

Fig. 8 The signal waveforms of FBG seismic sensor for human walking at different distances

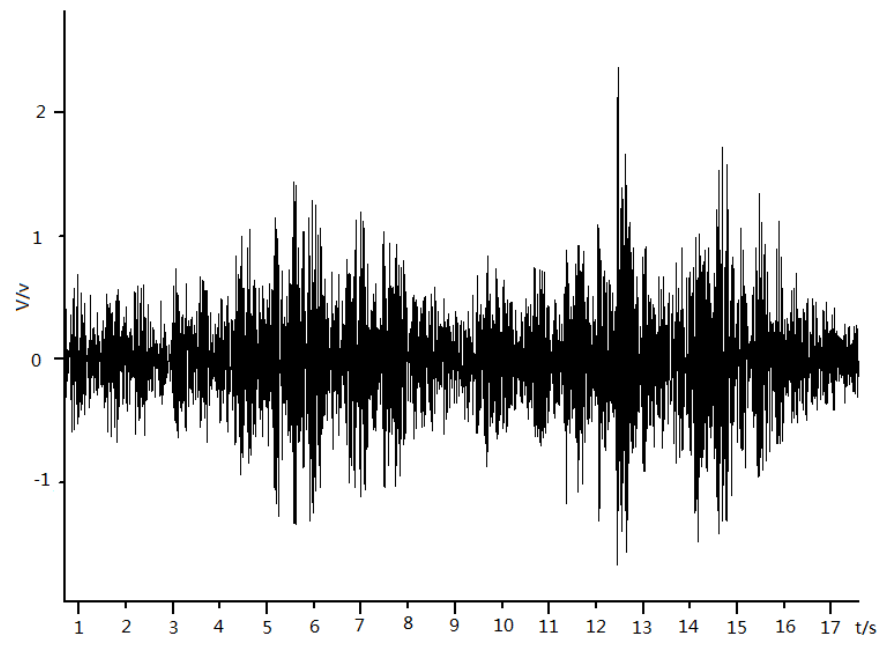

Fig.9 The signal waveform of FBG seismic sensor for small wheeled vehicle at $200 \mathrm{~m}$ distance

\section{CONCLUSION}

In this paper we report the development and test of a high sensitivity fiber Bragg grating (FBG) seismic sensor system for intrusion detection application. The performance of FBG seismic sensors are tested both in laboratory and the field test. FBG seismic sensor has significantly better performance in very small signal detection, and it is crucial for weak micro-seismic signal detection. The FBG seismic sensor has a maximum detection range of about 70 meters for human 
and about 200 meters for small wheeled vehicle. This work shows that using FBG technology to develop seismic sensor for intrusion detection is both advantageous and feasible.

\section{ACKNOWLEDGMENTS}

This work was supported by NSFC under Grant No. 61307101, National key research and development program No. 2017YFB0405505, the independent innovation major project of Shandong Province No. 2017XGC1609, and the outstanding youth program of Shandong Academy of Sciences.

\section{REFERENCES}

[1] Gregory H. Ames and Jason M. Maguire. Erbium Fiber Laser Accelerometer [J]. IEEE Sensors Journal, 7(4), (2007)557-561.

[2] Paulsson B N P, Toko J L, Thornburg J A, et al. Development of $300^{\circ} \mathrm{C}$ fiber optic seismic sensors for geothermal reservoir imaging and monitoring[J]. Transactions - Geothermal Resources Council, 2013:1043-1049.

[3] Zhihui Sun, Xiaohui Liu , Faxiang Zhang, et al.High Sensitivity fiber laser geophone array and field test analysis [J]. Measurement,79,(2016)216-221.

[4] Wentao Zhang, Fang Li, Yuliang Liu. Field test of an in-well fiber laser geophone array [C]. Proc. SPIE 8421(2B), (2012)1-4. 